Reprod. Nutr. Dévelop., 1980, 20 (6), 1855-1858.

\title{
Réalisation d'un réseau permettant la classification des structures histologiques de forme elliptique en fonction de leur taille
}

\author{
par J. C. MARIANA, J. BACHACOU *, M. TERRIOT
}

Station de Physiologie de la Reproduction, I. N. R. A. Nouzilly, 37380 Monnaie, France.

* Station de Biométrie, I. N. R. A., Champenoux, 54280 Seichamps, France

Summary. An array for classifying histological structures with an elliptic shape according to size.

An array of rectangular hyperbolas with the same center of symmetry is proposed for classifying microscopic particles with an elliptic section according to size and then determining their size. The measurement of the size of ovarian oocytes is used as an example.

En histologie quantitative, on est souvent conduit à établir la distribution des volumes ou des surfaces de particules microscopiques dont la forme est cylindrique ou ellipsoïdale. Selon la répartition ef le nombre des particules dans le tissu étudié, on pourra utiliser deux méthodes :

1) Si les particules sont grossièrement sphériques, uniformément réparties dans le tissu, on démontre que l'on peut reconstituer la distribution des volumes de ces particules à partir :

a) de la distribution des intercepts de ces particules avec un système de droites ;

b) de la distribution des surfaces des sections de ces particules par des plans (Serra, 1969 ; Solari, 1978).

On peut donc pratiquement reconstituer la distribution des volumes de particules sphériques à partir de la distribution des intercepts engendrés sur les sections circulaires des particules par un réseau de droites (Weibel et Gomez, 1962) superposé à l'image des sections des particules. Un microscope muni d'une table traçante (ASM, Leitz) permet de mesurer rapidement un grand nombre d'intercepts et d'établir la courbe de distribution.

L'application de cette méthode nécessite que le nombre de particules par unité de volume soit suffisamment grand, donc que le nombre de sections par portion de coupe observée soit suffisant pour permettre d'établir la distribution. 
2) Si les particules sont peu nombreuses dans le tissu, on peut mesurer directement la surface des sections circulaires de toutes les particules et établir la distribution des volumes des particules.

La méthode usuelle consiste à mesurer 2 diamètres orthogonaux de la section, soit directement sur une image projetée, soit au moyen d'un réticule placé dans l'oculaire d'un microscope, afin de déterminer la surface de l'ellipse; $a$ et $b$ sont les mesures des deux demi-axes principaux.

Dans la plupart des cas, une classification des surfaces en classes de faille apporte une précision suffisante pour calculer différents paramètres statistiques tels que moyenne, écart-type, et réaliser ensuite des tests de comparaison.

Nous avons réalisé dans ce but, un réseau utilisant la propriété suivante de l'ellipse.

Toutes les ellipses de même surface $S_{1}$ sont tangentes aux hyperboles équilatères d'équation $X Y= \pm S_{1} / 2 \pi$. On peut donc représenter les limites $S_{1}$ ef $S_{2}$ d'une classe de taille d'ellipses au moyen des 2 hyperboles équilatères d'équation $X Y= \pm S_{1} / 2 \pi$ et $X Y= \pm S_{2} / 2 \pi$.

On peut par exemple, prendre des limites de classe en progression géométrique (fig. 1). Le réseau représenté figure 1 a été réalisé au moyen d'un traceur de courbes (Calcomp à rouleau) sur lequel on a programmé l'équation de l'hyperbole équilatère ; le réseau ainsi tracé s'inscrivait dans un cadre carré de dimension $30 \mathrm{~cm} \times 30 \mathrm{~cm}$. Il peut être soit utilisé directement et superposé aux structures à mesurer, soit réduit par pholographie pour être monté dans un oculaire de microscope (comme n'importe quel réticule oculaire).

Le réseau initial a été réduit 15 fois en prenant comme film, le Kodalith super Ortho 2775 ; le contretype positif réalisé sur le même film a été ensuite découpé avec un emporte-pièce du diamètre de l'oculaire.

On réalise la mesure en superposant l'image de l'objet à celle du réseau (fig. 2).

Pour effectuer une mesure, on centre le réseau au centre approximatif de la section elliptique à mesurer et on l'oriente de façon à ce que les 2 axes du réseau coïncident approximativement avec ceux de l'ellipse. La mesure est alors immédiate : elle consiste à noter les numéros des branches d'hyperboles qui enveloppent l'ellipse Les numéros correspondent aux classes de surfaces considérées, délimitées par les branches d'hyperboles. Pour tester la validité des mesures par rapport à la méthode planimétrique standard, nous avons mesuré cent sections histologiques d'ovocytes de follicules d'ovaire de lapin ; l'image projetée de chaque section obtenue par un microscope à projection est directement planimétrée ef trois personnes ont encadré au moyen du réseau d'hyperboles les sections des ovocytes; sur un ensemble de 101 mesures on a relevé seulement 6 erreurs de classification (par ce réseau).

Ces erreurs étaient pour la plupart liées à une mauvaise définition de l'image de la pellucide entourant l'ovocyte. La surface moyenne des ovocytes mesurés direc-

FIG. 1. - L'ovocyfe de ce petit follicule ovarien appartient à la classe de taille limitée par les hyperboles $n^{\circ}$ 3-4 du réticule oculaire ; les limites de classes sont 243,48 ef $340,8 \mu^{2} \times 2400$.

FIG. 2. - Les branches des hyperboles équilatères 1, 2, 3 ... représentent les limifes de classe de taille. 


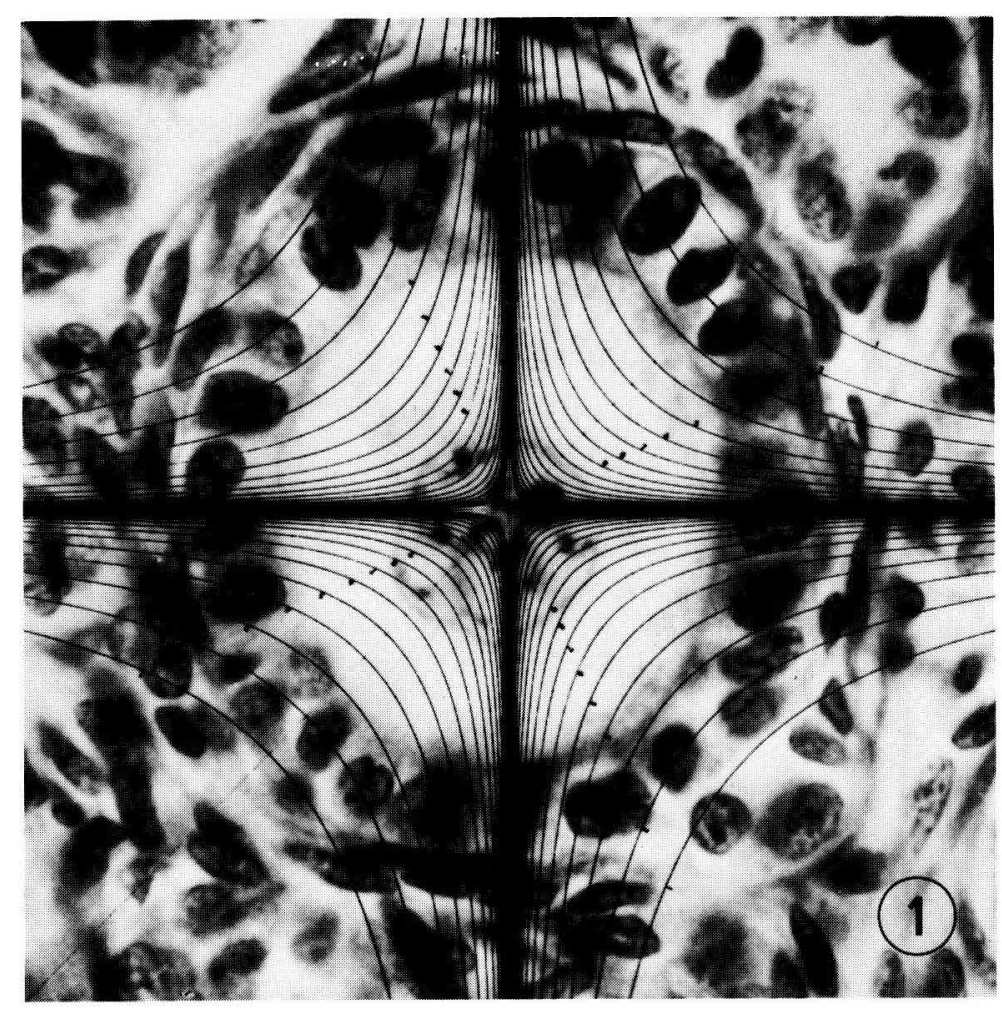

1857

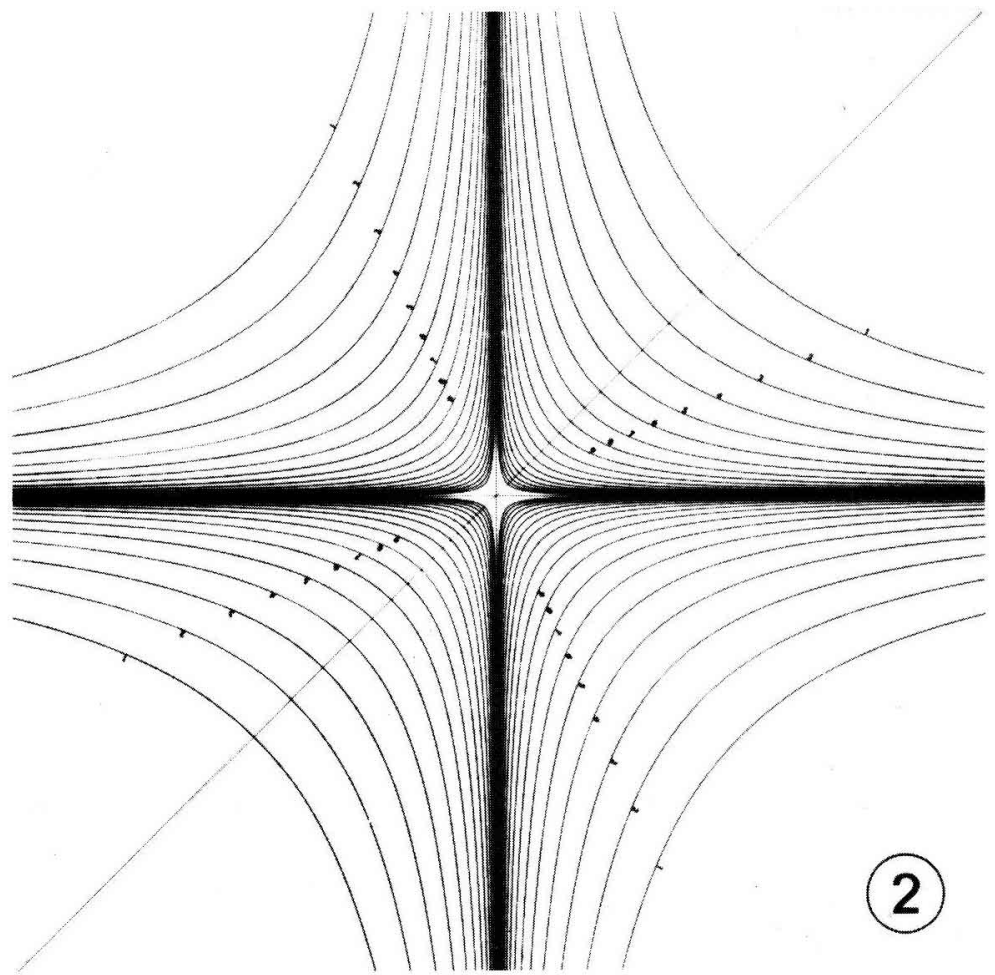


tement par planimétrie était $3451,78 \mu^{2}$ avec un écart type de $2557,08 \mu^{2}$ et les estimations de la surface moyenne el de l'écart type au moyen du réticule élaient respectivement $3465 \mu^{2}$ et $2537 \mu^{2}$; le réticule utilisé pour ce test était formé de 11 branches d'hyperboles délimifant des classes de taille en progression arithmétique de raison $960 \mu^{2}$.

La méthode est donc rapide à mettre en œuvre, suffisamment précise et reste valable lorsque la forme des sections s'écarte sensiblement de celle de l'ellipse.

Pour étalonner le réseau dans le cas où on réalise des agrandissements ou des réductions du réseau initial, on utilise comme ellipse particulière des cercles dont on mesure le rayon directement sur l'agrandissement du réseau initial ou avec un réticule objectif dans le cas où on réduit le réseau.

Reçu en septembre 1979 Accepté en juin 1980.

\section{References}

SERRA J., 1969. Introduction à la morphologie mathémafique. Cahiers Centre Morphol. mathémot. Fontainebleau, Fasc. 3.

SOLARI A., 1978. Etude quantitative d'organes ou de tissus. IV. Distribution de fréquence des diamètres de particules. Ann. Biol. anim. Biol. Biophys., 18, 1371-1392.

WEIBEL E. R., GOMEZ D. M., 1962. A principle for counting fissue structures on random section. J. appl. Physiol., 17, 343. 